\title{
A Novel Wireless Statistical Division Multiplexing Communication System and Performance Analysis
}

\author{
Wei Zhao ${ }^{* 1}$, Yuehong Shen ${ }^{1}$, Pengcheng Xu ${ }^{1}$, Jiangong Wang ${ }^{1}$, Zhigang Yuan ${ }^{1}$, \\ Yimin Wei ${ }^{1}$, Wei Jian ${ }^{1}$ and Hui $\mathrm{Li}^{2}$ \\ ${ }^{1}$ College of Communication Engineering, PLA University of Science and Technology, \\ Biaoying No.2, Yudao Street, Qinhuai District, 210007, Nanjing, China \\ ${ }^{2}$ PLA Troop78020, Kunming, Yunnan, China \\ *gmajyie@126.com
}

\begin{abstract}
This paper proposes a new wireless statistical division multiplexing (SDM) communication system, which is based on the two-input and two-output wireless communication experimental platform constructed and presented in our previous work. This novel system aims to transmit source signals simultaneously in the same frequency band over wireless channel and recovers the source signals at the receiver by utilizing the statistical characteristics of source signals and broadcasting characteristics of wireless channel. Therefore, the spectrum efficiency of SDM system is high compared to those of time division multiplexing (TDM), frequency division multiplexing (FDM), and code division multiplexing (CDM) systems, in which TDM, FDM and CDM signals are limited in time interval, frequency band and code. Taking advantage of the statistical properties of transmitted source signals, the SDM system can easily recover or retrieve them through the blind source separation (BSS) techniques at the receiver. This paper first introduces the system model of SDM briefly, but the main purpose is to analyze the performance of it including the relationship between the separation quality and the characteristics of source signals, the power of transmitters and separation algorithms. The performance validity and corresponding analysis are confirmed and performed through realistic experiments.
\end{abstract}

Keywords: wireless statistical division multiplexing communication system; statistically independent, blind source separation

\section{Introduction}

With the high-speed development of the modern society, exploitation and utilization of the natural resource, especially efficient exploitation and utilization of the finite natural resource has become one of the most important research fields [1]. The electromagnetic radio spectrum is a critical natural resource in informationized society. With each passing day, more people are subscribing to one of the plethora of wireless services currently available on the market. As a result of this rapid growth in the wireless services industry, the demand for additional bandwidth is steadily increasing despite the fact that frequency spectrum is a finite natural resource. Thus, to avoid a potential spectrum scarcity problem, both spectrum policy makers and wireless technology specialists are united in seeking solutions that would help efficiently exploit and utilize the finite natural resource in order to accommodate this rapid growth [2-4].

* Corresponding Author 
A communications resource represents the time and bandwidth that is available for communication signaling associated with a given system. For the efficient development of a communication system, it is important to plan out the resource allocation among system users, so that no communications resource is wasted, and so that the users can share the resource in an equitable manner [5-7]. The traditional ways of distribution the communications resource are as follows:

- Frequency division multiplexing (FDM). Specified sub-bands of frequency are allocated.

- Time division multiplexing (TDM). Periodically recurring time slots are identified.

- Code division multiplexing (CDM). Specified numbers of a set of orthogonal or nearly orthogonal spread spectrum code are allocated.

However, since TDM and FDM carve up the signaling dimensions orthogonally, there is a hard limit on how many orthogonal channels can be obtained. This is also true for CDM using orthogonal codes. If non-orthogonal codes are used, there is no hard limit on the number of channels that can be obtained. Hence, the TDM, FDM and CDM signal is limited in time interval, or frequency band or code [2-4].

Inspired by our previous work ${ }^{1}$, in which we have constructed a simple two-input and two-output wireless communication system, a novel wireless statistical division multiplexing (SDM) communication system is proposed. The transmitted source signals are required to be statistically independent, which are transmitted through the radio frequency (RF) antennas. At the receivers, they are recovered through the blind source separation (BSS) algorithms [8]-[10] by utilizing the statistical characteristics of them and broadcasting characteristics of wireless channel. Therefore, the spectrum efficiency of our proposed system is high compared to those of TDM, FDM, and CDM systems, and the new SDM system is very simple and easy to be implemented for it is regardless of considering the limitation of time interval, frequency band and code as the traditional ones do. Besides, the main work in this paper is to analyze the performance of this new system based on our previous work, which mainly deals with the influence of the diversity and power of transmitted source signals and separation algorithms on the separation quality. Real-life experiments are performed to validate the performance of our SDM system.

\section{SDM Model}

The system model of SDM is shown in Figure 1. For it has been presented in our previous work, we will introduce it briefly in this paper. This model includes two RF antennas as transmitters and two receiving antennas as receivers. The source signals are comprised of I and Q components. And the RF and local frequencies are different from each other, but we assume they are same for simplicity in this paper, i.e., $\omega_{1}=\omega_{2}=\omega_{3}=\omega_{4}=\omega_{0}$. And we don't consider the synchronous and carrier frequency offset problems, which will be included in our future work.

${ }^{1}$ The two-input and two-output wireless communication system presented in our previous work has been submitted to another conference. The main work in it shows theoretically that the information content of all the source signal inputs 

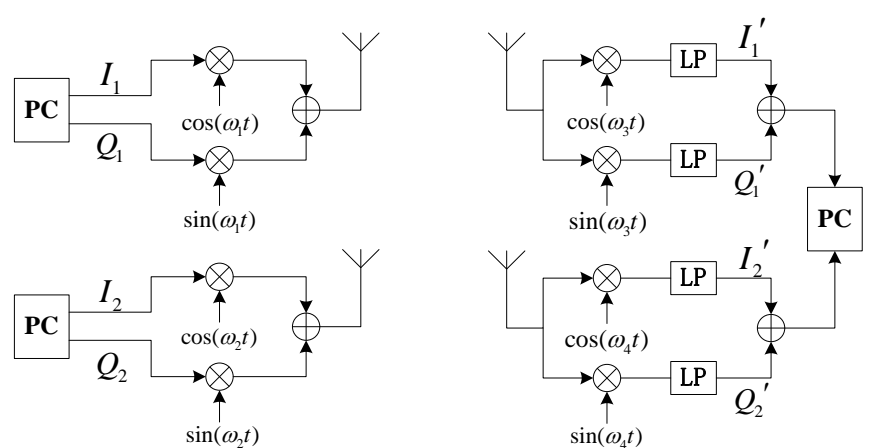

Figure 1 The SDM System Model

The two input source signals are denoted by

$$
\begin{aligned}
& s_{1}=I_{1}+Q_{1} i \\
& s_{2}=I_{2}+Q_{2} i
\end{aligned}
$$

After modulating they are expressed as

$$
\begin{aligned}
& s_{1}^{\prime}=I_{1} \cos \omega_{0} t+Q_{1} \sin \omega_{0} t \\
& s_{2}^{\prime}=I_{2} \cos \omega_{0} t+Q_{2} \sin \omega_{0} t
\end{aligned}
$$

The received signals result from the mixture of sources, i.e.,

$$
\left(\begin{array}{l}
x_{1} \\
x_{2}
\end{array}\right)=\mathbf{A}\left(\begin{array}{l}
s_{1}^{\prime} \\
s_{2}^{\prime}
\end{array}\right)=\left(\begin{array}{ll}
a_{11} & a_{12} \\
a_{21} & a_{22}
\end{array}\right)\left(\begin{array}{l}
s_{1}^{\prime} \\
s_{2}^{\prime}
\end{array}\right)
$$

Where A denotes the wireless channel. For simplicity, we only consider the linear mixing case in this paper, i.e., the elements of $\mathbf{A}$ are constant. In realistic experiments, we set the transmitting and receiving antennas close to each other so that the wireless mixing channel can be treated as approximately linear.

In order to analyze the performance of this novel system, we should make sure the wireless channel as simple as possible, in case that the multi-path and time delay effects become the main influence, which will make this system very complex and hard to distinguish the source signals form interferences. With respect to more complex channels such as the convolution and nonlinear mixing channels, we will address in our following research. However, note that the interferences from outside environment like noise still exist, even though we don't take them into consideration, which can be obviously observed in the following experimental section and they can be ignored to some extent. The mixing signals are

$$
\begin{aligned}
x_{1} & =a_{11}\left(I_{1} \cos \omega_{0} t+Q_{1} \sin \omega_{0} t\right)+a_{12}\left(I_{2} \cos \omega_{0} t+Q_{2} \sin \omega_{0} t\right) \\
& =\left(a_{11} I_{1}+a_{12} I_{2}\right) \cos \omega_{0} t+\left(a_{11} Q_{1}+a_{12} Q_{2}\right) \sin \omega_{0} t \\
x_{2} & =a_{21}\left(I_{1} \cos \omega_{0} t+Q_{1} \sin \omega_{0} t\right)+a_{22}\left(I_{2} \cos \omega_{0} t+Q_{2} \sin \omega_{0} t\right) \\
& =\left(a_{21} I_{1}+a_{22} I_{2}\right) \cos \omega_{0} t+\left(a_{21} Q_{1}+a_{22} Q_{2}\right) \sin \omega_{0} t
\end{aligned}
$$

After demodulating, the received signals can be presented as

$$
\begin{aligned}
x_{1}^{\prime} & =\left\{\left(a_{11} I_{1}+a_{12} I_{2}\right) \cos \omega_{0} t+\left(a_{11} Q_{1}+a_{12} Q_{2}\right) \sin \omega_{0} t\right\} \cos \omega_{0} t \\
& =\frac{1}{2}\left(a_{11} I_{1}+a_{12} I_{2}\right)+\frac{1}{2}\left(a_{11} I_{1}+a_{12} I_{2}\right) \cos 2 \omega_{0} t+\frac{1}{2}\left(a_{11} Q_{1}+a_{12} Q_{2}\right) \sin 2 \omega_{0} t
\end{aligned}
$$




$$
\begin{aligned}
x_{1}^{\prime \prime}= & \left\{\left(a_{11} I_{1}+a_{12} I_{2}\right) \cos \omega_{0} t+\left(a_{11} Q_{1}+a_{12} Q_{2}\right) \sin \omega_{0} t\right\} \sin \omega_{0} t \\
= & \frac{1}{2}\left(a_{11} I_{1}+a_{12} I_{2}\right) \sin 2 \omega_{0} t+\frac{1}{2}\left(a_{11} Q_{1}+a_{12} Q_{2}\right)+\frac{1}{2}\left(a_{11} Q_{1}+a_{12} Q_{2}\right) \cos 2 \omega_{0} t \\
x_{2}^{\prime} & =\left\{\left(a_{21} I_{1}+a_{22} I_{2}\right) \cos \omega_{0} t+\left(a_{21} Q_{1}+a_{22} Q_{2}\right) \sin \omega_{0} t\right\} \cos \omega_{0} t \\
& =\frac{1}{2}\left(a_{21} I_{1}+a_{22} I_{2}\right)+\frac{1}{2}\left(a_{21} I_{1}+a_{22} I_{2}\right) \cos 2 \omega_{0} t+\frac{1}{2}\left(a_{21} Q_{1}+a_{22} Q_{2}\right) \sin 2 \omega_{0} t \\
x_{2}^{\prime \prime} & =\left\{\left(a_{21} I_{1}+a_{22} I_{2}\right) \cos \omega_{0} t+\left(a_{21} Q_{1}+a_{22} Q_{2}\right) \sin \omega_{0} t\right\} \sin \omega_{0} t \\
& =\frac{1}{2}\left(a_{21} I_{1}+a_{22} I_{2}\right) \sin 2 \omega_{0} t+\frac{1}{2}\left(a_{21} Q_{1}+a_{22} Q_{2}\right)+\frac{1}{2}\left(a_{21} Q_{1}+a_{22} Q_{2}\right) \cos 2 \omega_{0} t
\end{aligned}
$$

After low-pass filtering, the above signals are as follows, denoted by

$$
\begin{array}{ll}
I_{1}^{\prime}=\frac{1}{2}\left(a_{11} I_{1}+a_{12} I_{2}\right) & Q_{1}^{\prime}=\frac{1}{2}\left(a_{11} Q_{1}+a_{12} Q_{2}\right) \\
I_{2}^{\prime}=\frac{1}{2}\left(a_{21} I_{1}+a_{22} I_{2}\right) & Q_{2}^{\prime}=\frac{1}{2}\left(a_{21} Q_{1}+a_{22} Q_{2}\right)
\end{array}
$$

Then the relationship between the $I / Q$ components of the original sources and the received signals after demodulating and filtering are shown as

$$
\begin{aligned}
& \left(\begin{array}{c}
I_{1}^{\prime} \\
I_{2}^{\prime}
\end{array}\right)=\left(\begin{array}{ll}
a_{11}^{\prime} & a_{12}^{\prime} \\
a_{21}^{\prime} & a_{22}^{\prime}
\end{array}\right)\left(\begin{array}{l}
I_{1} \\
I_{2}
\end{array}\right) \\
& \left(\begin{array}{l}
Q_{1}^{\prime} \\
Q_{2}^{\prime}
\end{array}\right)=\left(\begin{array}{ll}
a_{11}^{\prime} & a_{12}^{\prime} \\
a_{21}^{\prime} & a_{22}^{\prime}
\end{array}\right)\left(\begin{array}{l}
Q_{1} \\
Q_{2}
\end{array}\right)
\end{aligned}
$$

Where $\left(\begin{array}{ll}a_{11}^{\prime} & a_{12}^{\prime} \\ a_{21}^{\prime} & a_{22}^{\prime}\end{array}\right)=\frac{1}{2}\left(\begin{array}{ll}a_{11} & a_{12} \\ a_{21} & a_{22}\end{array}\right)$.

Then the two corresponding output signals are in the form of

$$
\begin{aligned}
x_{1}^{\prime \prime \prime} & =a_{11}^{\prime} I_{1}+a_{12}^{\prime} I_{2}+\left(a_{11}^{\prime} Q_{1}+a_{12}^{\prime} Q_{2}\right) i=a_{11}^{\prime}\left(I_{1}+Q_{1} i\right)+a_{12}^{\prime}\left(I_{2}+Q_{2} i\right) \\
& =a_{11}^{\prime} s_{1}+a_{12}^{\prime} s_{2} \\
x_{2}^{\prime \prime \prime} & =a_{21}^{\prime} I_{1}+a_{22}^{\prime} I_{2}+\left(a_{21}^{\prime} Q_{1}+a_{22}^{\prime} Q_{2}\right) i=a_{21}^{\prime}\left(I_{1}+Q_{1} i\right)+a_{22}^{\prime}\left(I_{2}+Q_{2} i\right) \\
& =a_{21}^{\prime} s_{1}+a_{22}^{\prime} s_{2}
\end{aligned}
$$

Where the signals $x_{1}^{\prime \prime \prime}$ and $x_{2}^{\prime \prime \prime}$ are used in the BSS separation algorithm to recover transmitted sources.

\section{Separation Algorithm}

According to the statistically properties of source signals, the separation problem of our SDM system can easily find solutions through the blind source separation (BSS) techniques [8-10], which corresponds to the independent component analysis (ICA) when the linear and instantaneous case is considered. In this paper, we choose several classical complex-valued ICA algorithms [10-11], which are gradient and fast fixed-point algorithms based on kurtosis and negentropy respectively. With respect to the separation system, a separation matrix is chosen as 


$$
\mathbf{W}=\left(\mathbf{w}_{1}, \mathbf{w}_{2}\right)=\left(\begin{array}{ll}
w_{11} & w_{12} \\
w_{21} & w_{22}
\end{array}\right)
$$

Where the elements of $\mathbf{W}$ are complex-valued.

Then the separation process is denoted by

$$
\left(\begin{array}{l}
y_{1} \\
y_{2}
\end{array}\right)=\mathbf{W}^{H} \mathbf{x}=\left(\begin{array}{ll}
w_{11}{ }^{*} & w_{21}{ }^{*} \\
w_{12}{ }^{*} & w_{22}{ }^{*}
\end{array}\right)\left(\begin{array}{l}
x_{1}^{\prime \prime \prime} \\
x_{2}^{\prime \prime \prime}
\end{array}\right)
$$

Where the recovered signals can be treated as the approximate estimation of the sources. $\mathbf{W}^{H}$ stands for the Hermitian of $\mathbf{w}$, that is $\mathbf{w}$ is transposed and conjugated. Then, $\mathbf{y}(t)=\mathbf{W}^{H} \mathbf{x}(t)$ is the recovered estimation of source signals up to some ambiguities, namely ordering and scaling factors, which are the inherent properties of BSS or ICA [11]. However, the ambiguities are, fortunately, insignificant in most applications, so they are out of the scope of this paper.

It is well accepted that the mixture signals are prewhitened, with mean value eliminated, before separation for ICA algorithms in the linear and instantaneous case in general. Then, the mentioned ICA algorithms are as follows:

$$
\begin{aligned}
& \mathbf{w}^{\prime}\left.=\mathbf{w}-\lambda E\left\{\mathbf{x}\left(\mathbf{w}^{H} \mathbf{x}\right)^{*}\left|\mathbf{w}^{H} \mathbf{x}\right|^{2}\right)\right\} \\
& \mathbf{w}^{\prime}=E\left\{\mathbf{x}\left(\mathbf{w}^{H} \mathbf{x}\right)^{*}\left|\mathbf{w}^{H} \mathbf{x}\right|^{2}\right\}-3 E\left\{\left|\mathbf{w}^{H} \mathbf{x}\right|^{2}\right\} \mathbf{w} \\
& \mathbf{w}^{\prime}\left.\left.=\mathbf{w}-\lambda E\left\{\mathbf{x}\left(\mathbf{w}^{H} \mathbf{x}\right)^{*} g\left(\mathbf{w}^{H} \mathbf{x}\right)\left(\mathbf{w}^{H} \mathbf{x}\right)^{*}\right)\right\}\right\} \\
&\left.\left.\left.\left.\mathbf{w}^{\prime}=E\left\{\mathbf{x}\left(\mathbf{w}^{H} \mathbf{x}\right)^{*} g\left(\mathbf{w}^{H} \mathbf{x}\right)\left(\mathbf{w}^{H} \mathbf{x}\right)^{*}\right)\right\}\right\}-E\left\{g\left(\mathbf{w}^{H} \mathbf{x}\right)\left(\mathbf{w}^{H} \mathbf{x}\right)^{*}\right)+\left(\mathbf{w}^{H} \mathbf{x}\right)\left(\mathbf{w}^{H} \mathbf{x}\right)^{*} g^{\prime}\left(\mathbf{w}^{H} \mathbf{x}\right)\left(\mathbf{w}^{H} \mathbf{x}\right)^{*}\right)\right\} \mathbf{w}
\end{aligned}
$$

Where (28) and (29) are gradient and fast fixed-point algorithms based on kurtosis [10], respectively. $\lambda$ denotes the iteration step size. And (30) and (31) are corresponding nengentropy-based algorithms, separately. $g$ and $g^{\prime}$ are the derivatives of the nonlinear function $G$ and $g$ [11], respectively. In this paper, we choose $G(y)=\log (a+y)$, and then $g(y)=\frac{1}{a+y}, \quad g^{\prime}(y)=-\frac{1}{(a+y)^{2}}$. Note that all iteration processes are used to extract one source signal at a time and the sources are extracted one by one in a deflation manner. To prevent different one-dimensional optimization converging to the same maxima, a Gram-Schmidt-like decorrelation scheme [11] is adopted in our separation process.

\section{Experimental Results and Performance Analysis}

First of all, in order to eliminate the nonlinear distortion caused by the power amplifier in the transmitters, we take two E4438C [12] as the transmitters, which can send radio signals in the form of single, AM, BPSK, speech and so on. Then, at the receivers, we use the USRP with GUN Radio [13] device to receive the RF signals.

Secondly, to be able to make the transmitted source signals as statistically independent as possible, we set the distance between two transmitters about ten meters. And we set the distance between transmitters and receivers about five meters so as to ensure the wireless channel as approximately linear as possible. And moreover, it is well accepted that most of the communication signals are uncorrelated and nonguanssian in general. 
Finally, we measure the separation quality between retrieved signals and the received signals in the case that only one transmitter send source signal. In other words, we just compare the recovered signals with the received signals when only one transmitter works. More precisely, we only care about the successfully separation of source signals in this paper, and the traditional measurements and methods to eliminate the interference, noise, frequency selective fading and so on are out of scope of this paper.

\subsection{Separability}
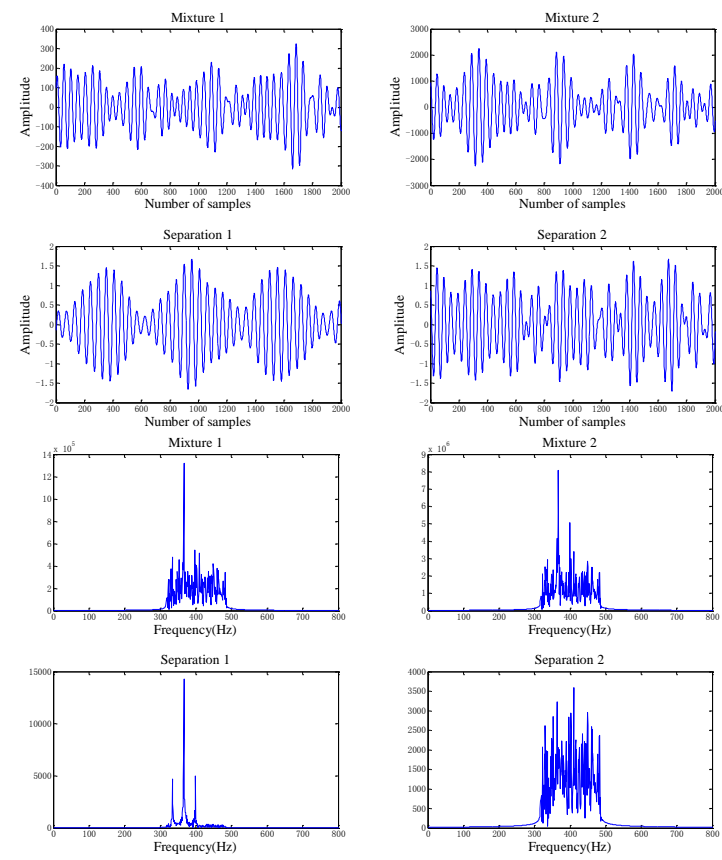

(a)
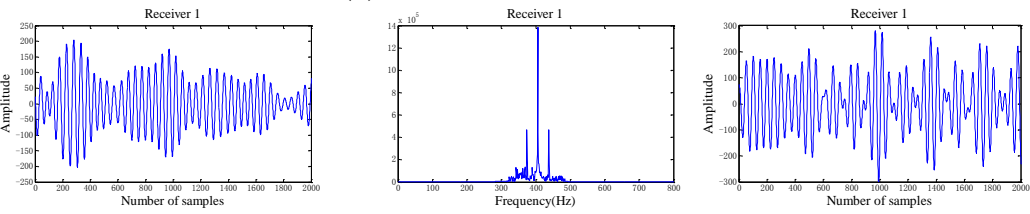

(b)
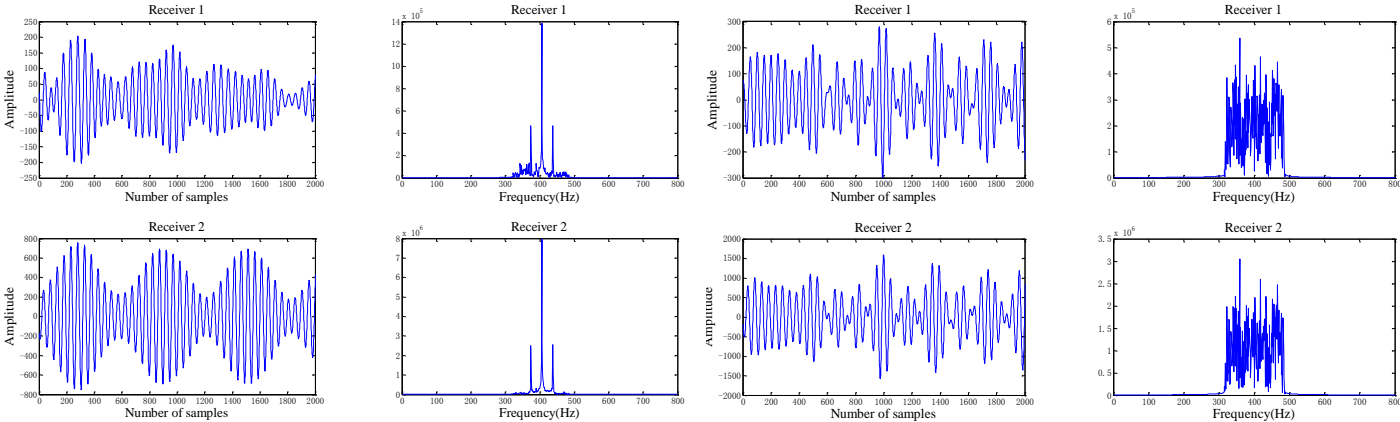

(c)

(d)

Figure 2. Two Independent Sources: AM and BPSK Signals. (a) Mixture and Separation Signals In Time Domain. (b) Mixture and Separation Signals in Frequency Domain. (c) Received AM Signals with only one Transmitter Working. (d) Received BPSK Signals with only one Transmitter Working

As shown in Figure 2, we use AM and BPSK signals as sources, for which the carrier frequency is set $30 \mathrm{MHz}$ and the power of two transmitters are $0 \mathrm{dBm}$. And the fast fixedpoint algorithm based on nengentropy in (31) is chosen as the separation algorithm. This experiment is just used to validate the separability of our proposed SDM system. Because the 
corresponding analysis in detail has been included in our previous work, we just give a brief introduction in this paper.

Compared (a) with (b), we can obviously see that the source signals are recovered. Then, comparing the separation 1 in (a) with receiver 2 in (c) in time domain or separation 1 in (b) with receiver 2 in (c) in frequency domain, it is clearly that the AM source signal is successfully recovered in the way we talked above. And the BPSK case is truly same when comparing the separation 2 in (a) with receiver 2 in (d) in time domain or separation 1 in (b) with receiver 2 in (d) in frequency domain. Therefore, our proposed SDM system works well with signals transmitted and received simultaneously in the same frequency band over wireless channel, which is the main advantage of SDM over traditional ones.

\subsection{Performance Analysis with Different Source Signals}

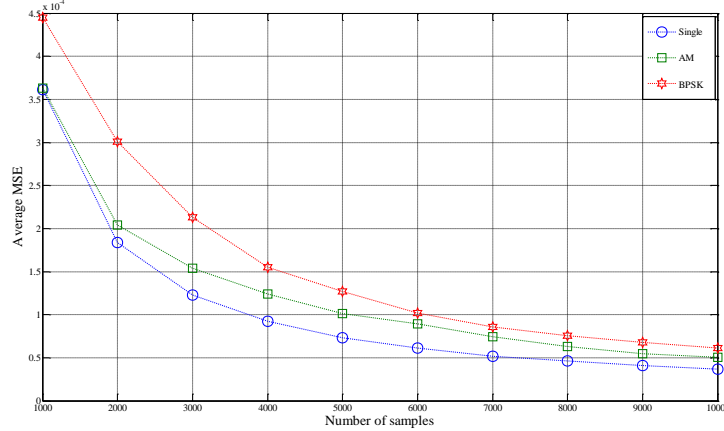

(a)

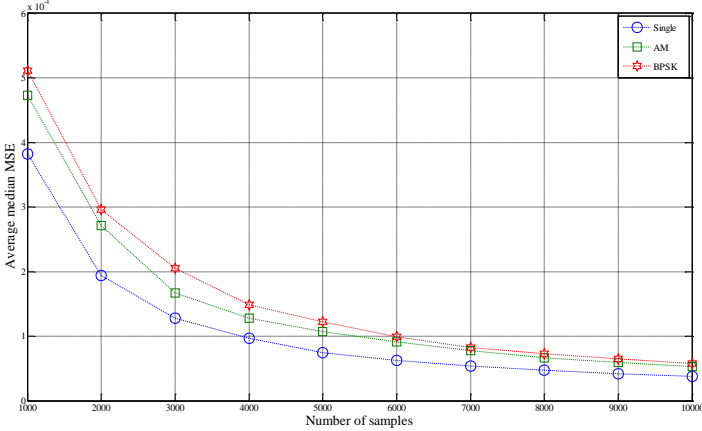

(b)

Figure 3 Average MSE and Median MSE between Sources And Separations for Different Kind Of Source Signals Averaged over 100 Independent Runs. (a) Average MSE for Single, AM and BPSK Signals Averaged Over 100 Independent Runs. (b) Average Median MSE for Single, AM and BPSK Signals Averaged Over 100 Independent Runs

In this section, we perform a realistic experiment to validate the performance of the SDM system with different kind of source signals, which are single, AM and BPSK signals respectively. The experimental result is shown in Fig. 3. (a) and (b) show the performance comparison, in which average MSE denotes the mean of mean square error and average median MSE denotes the median value of mean square mean error between sources and separations averaged over 100 independent runs. In this experiment, the carrier frequency is set $30 \mathrm{MHz}$, the fast fixed-point algorithm based on the nengentropy shown in (31) is chosen as the separation algorithm and the transmitted signal power is set $0 \mathrm{dBm}$.

As shown in Figure 3, it is clear that different sources result in different performance, i.e. the MSE and median MSE. More precisely, single singles performs better than AM signals, and BPSK is the worst, which is caused by their characteristics. It is well accepted that single signals are simpler than AM, and BPSK is the most complicated. Therefore, it could be drawn a conclusion, to a certain extent, that the more source signal complex, the worse the performance is. Of course, we only perform three simple signals, and more complicated signals will be considered in our future work such as QPSK, OFDM, speech signals. But this experiment reveals the character of our proposed system for different signals to some extent, which provides reference for our future work. 


\subsection{Performance Analysis with Different Transmitted Power}

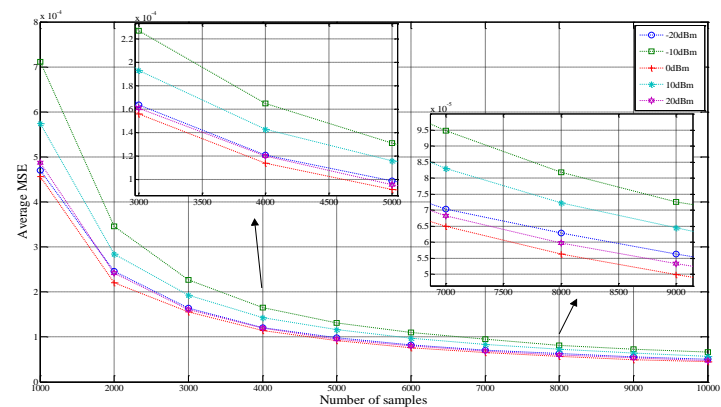

(a)

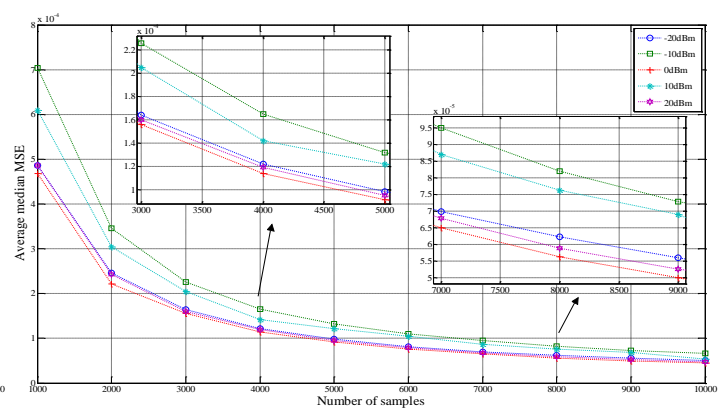

(b)

Figure 4 Average MSE and Median MSE between Sources and Separations for Different Transmitted Power Of Source Signal Averaged Over 100 Independent Runs. (a) Average MSE for $-20 \mathrm{dBm},-10 \mathrm{dBm}, 0 \mathrm{dBm}, 10 \mathrm{dBm}$ and $20 \mathrm{dBm}$ Averaged Over 100 Independent Runs. (b) Average Median MSE for -20dBm, $10 \mathrm{dBm}, 0 \mathrm{dBm}, 10 \mathrm{dBm}$ and $20 \mathrm{dBm}$ Averaged Over 100 Independent Runs

In this experiment, we conduct an experiment to validate the performance of the SDM system with different transmitted source signal power, i.e., $-20 \mathrm{dBm},-10 \mathrm{dBm}, 0 \mathrm{dBm}, 10 \mathrm{dBm}$, $20 \mathrm{dBm}$. In this experiment, the carrier frequency is set $30 \mathrm{MHz}$, the source signal is chosen to be single signal, the gradient algorithm based on kurtosis with the step size $\lambda=0.1$ shown in (28) is use as the separation algorithm. As shown in (a) and (b) in Fig. 4, different transmitted power leads to different separation quality, in which $0 \mathrm{dBm}$ provides the best performance and $-10 \mathrm{dBm}$ provides the worst.

However, note that the performance curves with different transmitted power are very close to each other, especially when the number of samples becomes large. Therefore, the feeling that the more the transmitted power, the better the performance will be may not true in a way. At least, in our experiment, a conclusion is drawn that an appropriate choice of transmitted power may be best. Considering the some limitations of our experiment, we can't give a precise conclusion and more corresponding work will be done in the following days. But the experimental result provides us with some reference, which is meaningful and helpful. The corresponding following work will include the performance with different transmitted power and separation algorithms for different source signals, especially when the mixing system is more complicated such as the convolution channel and nonlinear channel.

\subsection{Performance Analysis with Different Separation Algorithms}

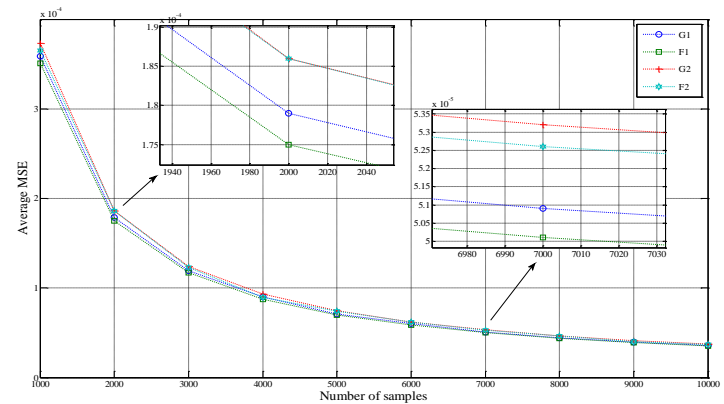

(a)

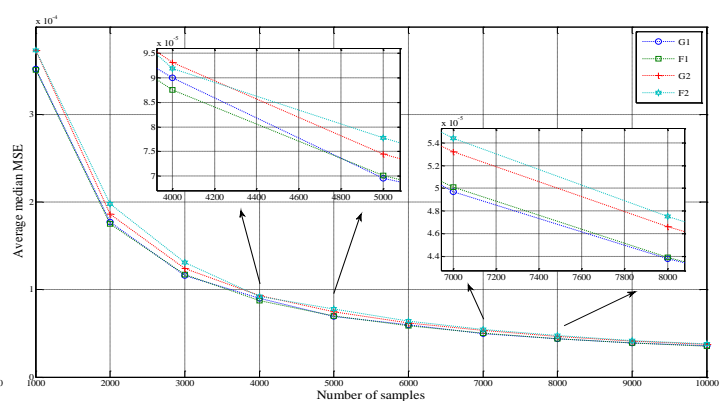

(b) 


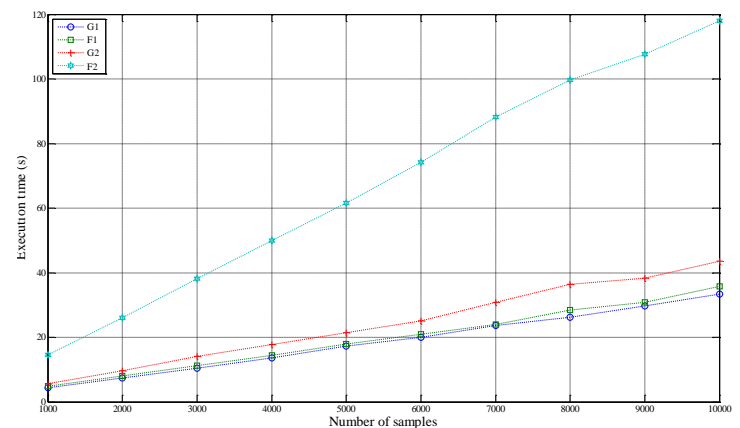

(c)

Figure 5 Average MSE and Median MSE between Sources and Separations for Different Separation Algorithms Averaged Over 100 Independent Runs, and Execution Time of Recovering all Source Signals For 100 Independent Runs. (a) Average MSE for G1, F1, G2 and F2 Averaged Over 100 Independent Runs. (b) Average median MSE for G1, F1, G2 and F2 Averaged Over 100 Independent Runs. (c) Execution Time Of Recovering all Source Signals for 100 Independent Runs

In this experiment, we consider the performance of the SDM system with different separation algorithms. As shown in (28), (29), (30) and (31), they are gradient and fast fixedpoint algorithms based on kurtosis and nengentropy, which are denoted by G1, F1, G2 and F2, respectively. More specifically, G1 denotes the gradient algorithm based on kurtosis, F1 denotes the fast fixed-point one based on kurtosis, G2 denotes the gradient one based on negentropy, and F1 denotes the fast fixed-point one based on negentropy. In this experiment, the carrier frequency is set $30 \mathrm{MHz}$, the source signal is chosen to be AM signals, the transmitted power is set $0 \mathrm{dBm}$. As for the gradient ones, the step size $\lambda=0.1$.

As illustrated in (a), (b) in Figure 5, different separation algorithms provide different separation quality. But note that the performance provided by them is very close to each other, which means that they are approximately identical. Compared (a) with (b), we can see that the value of MSE and median MSE is very close, which means that all the corresponding algorithms are very stable. As for (c) in Figure 5, the execution time means the time of recovering all sources for 100 independent runs, which confirms that they are all efficient enough. Note that the step size we choose here is a litter large; and when it is set smaller like $\lambda=0.01$, the corresponding execution time will be more. However, we only consider four simple algorithms, which may not sufficient but can be reference for our following work. Therefore, we will conduct experiments with more different kinds of algorithms that are appropriate for more complicated environment such as channels with multi-path, time delay and noise.

\section{Conclusion}

In this paper, we construct a novel wireless statistical division multiplexing communication system, which transmits source signals simultaneously in the same frequency band over wireless channel and recovers the source signals at the receiver by utilizing the statistical characteristics of source signals and broadcasting characteristics of wireless channel. The main advantage of our system consists in the high spectrum efficiency over the traditional ones such as TDM, FDM and CDM. The performance of this SDM system is validated through realistic experiments, in which the restored 
signals are compared with the received signals with one transmitter working. And simple performance analysis is conducted mainly considering different source signals, transmitted power and separation algorithms. Our future work includes the performance analysis for convolution and nonlinear channels with the multi-path, time-delay interferences and noise, which includes the choice of sources, transmitted power, separation algorithm, the distance between transmitters and so on. In a word, the simple linear SDM system constructed in this paper is a good beginning.

\section{Acknowledgements}

This work is supported by the Provincial Natural Science Foundation of Jiangsu province in China under Grant No. BK2011117 and by the National Natural Science Foundation of China under Grant No. 61172061.

\section{References}

[1] P. Jung, P. W. Baier and A. Steil, "Advantages of CDMA and spread spectrum techniques over FDMA and TDMA in cellular mobile radio applications", IEEE, Transactions on Vehicular Technology, (1993), pp. 357-364.

[2] T. S. Rappaport, "Wireless Communications - Principles and Practice", IEEE Press, (1996).

[3] W. Stallins, "Wireless Communications and Networks', 2nd Ed., Prentice Hall, (2005).

[4] A. Goldsmith, "Wireless Communications, Cambridge University Press", (2005).

[5] Q. Gao, H. M. Jonathan, G. Chen and Y. B. Hua, "Castellation design for a multicarrier Optical wireless communication channel”, IEEE Transactions on Communication, vol. 62, no. 1, (2014), pp. 214-225.

[6] P. J. Smith, P. A. Dmochowski, M. Chiani and A. Giorgetti, "On the number of independent channels in multi-antenna systems", IEEE Transactions on Wireless Communication, vol. 13, no. 1, (2014).

[7] L. H. Liu, H. Feng, T. Yang and B. Hu, "MIMO-OFDM wireless channel prediction by Exploiting spatialtemporal correlation", IEEE Transactions on Wireless Communication, vol. 13, no. 1, (2014).

[8] A. Yeredor, "Performance Analysis of the strong uncorrelating transformation in blind separation of complex-valued sources", IEEE Transactions on signal processing, vol. 60, no. 1, (2012), pp. 478-483.

[9] M. S. Alireza and D. R. Bhaskar, "An ICA-SCT-PHD filter approach for tracking and separation of unknown time-varying number of sources", IEEE Transactions on audio, speech, and language processing, vol. 21, no. 4, (2013), pp. 828-841.

[10] A. Hyvärinen and E. Oja, "A fast fixed-point algorithm for independent component Analysis", Neural Networks, vol. 10, no. 3, (1999), pp. 626-634.

[11] E. Bingham and A. Hyvärinen, "A fast fixed-point algorithm for independent component analysis of complex valued signals", International Journal of Neural System, vol. 10, no. 1, (2000), pp. 1-8.

[12] http://www.home.agilent.com.

[13] http://gnuradio.org/redmine/projects/gnuradio/wiki/USRP. 